\title{
A STUDY OF DRUG-RELATED PROBLEM IN UROLOGIC PATIENTS: A COHORT PROSPECTIVE STUDY
}

\author{
ANA KHUSNUL FAIZAH ${ }^{1 *}$, RASWITA DINIYA ${ }^{2}$ \\ ${ }^{1}$ Department of Pharmacy, Hang Tuah University, Indonesia. ${ }^{2}$ Department of Pharmacy, dr. Soetomo Teaching Hospital, Indonesia. \\ Email: anakhusnulfaizah@gmail.com.
}

Received: 4 November 2016, Revised and Accepted: 24 January 2017

\section{ABSTRACT}

Objective: Drug Related problems (DRPs) are the clinical domain of the pharmaceutical care practitioner. The purpose of identifying drug therapy problems is to help patients on achieving their goals of therapy and realizing the best possible outcomes from drug therapy. The purpose of this study was to determine the number of DRPs and to classify DRPs that occurred in post-operative patients from urology surgery ward.

Methods: A 3-month prospective study was conducted by considering 62 hospitalized post-operative patients from urology surgery ward at the Teaching Hospital in Surabaya, East Java, Indonesia. Clinical pharmacists were involved in medical rounds to identify DRPs.

Results: From 37 patients, 81 DRPs were identified in this study. About 20 patients were considered to have more than one DRP. The most common identified DRPs were overdosage (38\%), drug use without indication (20\%), improper drug (11\%), drug interaction (10\%), untreated indication (9\%), adverse drug reaction (7\%), failure to receive drug (4\%), and subtherapeutic drugs (1\%). The clinical pharmacists were involved in doing dose adjustment, monitoring, evaluating drug discontinuation, doing drug substitution and additional therapy, counseling to patients, increasing dose, and referring to prescriber.

Conclusion: The most identified DRPs were overdosage, drug use without indication, and improper drug. Clinical pharmacist's intervention was required to identify, prevent, resolve DRPs, and assist patient on achieving their goals of therapy and improving their quality of life.

Keywords: Drug therapy problems, Clinical pharmacist, Drug therapy, Drug interactions, Adverse drug reactions.

(C) 2017 The Authors. Published by Innovare Academic Sciences Pvt Ltd. This is an open access article under the CC BY license (http://creativecommons. org/licenses/by/4. 0/) DOI: http://dx.doi.org/10.22159/ajpcr.2017.v10s2.19472

\section{INTRODUCTION}

The purpose of pharmaceutical care is to achieve defined outcomes that can improve patients' quality of life by identifying, preventing, and resolving drug-related problems (DRPs) [1,2].

DRP is defined as an event that may potentially affect patient's health outcomes. DRPs can be initially found from prescribing to dispensing stage and may be caused by the lack of follow-up and reassessment of therapeutic outcomes [3]. DRPs can be classified in many classification systems, including the ABC of DRPs, American Society of Hospital Pharmacists system, a study by Cipolle et al., Hanlon et al., Hepler and Strand, and Krska et al. system, Granada consensus, Mackie classification, National Coordinating Council for Medication Error Reporting and Prevention, Pharmaceutical Care Network Europe classification, performance assessment system coding system, problem-intervention documentation SHB-SEP classification, and Westerlund classification [4].

Clinical pharmacists have to ensure the use of appropriate medication in patients through evidence-based medicine and identification, prevention, and resolving of DRPs to achieve goals of therapy, and improve patient's quality of life.

In Indonesia, there are only limited studies related to DRPs, especially conducted by a clinical pharmacist at the Teaching Hospital in Surabaya, East Java, Indonesia. The aim of this study was to determine the number of DRPs and to classify DRPs that occurred in post-operative patients from urology surgery ward.

\section{METHODS}

\section{Study population}

We conducted a cohort prospective observational study for 3 months, including 62 hospitalized post-operative patients. We conducted a cohort pros[ective observational study for 3 months, including 62 hospitalized post-operative patients. The study was approved by the research ethics commitee of Teaching Hospital (approval number, 345/ Panke/ VIII). Individuals were included in the study if they were hospitalized in the urology surgey ward of the Teaching Hospital in Surabaya. Clinical pharmacist was involved in medical rounds to identify DRPs and assess the characteristics of patients DRPs. Individuals were included in the study if they were hospitalized in the urology surgery ward of the Teaching Hospital in Surabaya. Clinical pharmacist was involved in medical rounds to identify DRPs and assess the characteristics of patients DRPs.

\section{Definitions}

DRP was defined as an event or circumstance that involves a patient's drug treatment, which might potentially interfere the achievement of an optimal outcome. The classifications of DRPs were based on the Hepler and Strand system.

- Untreated indications. The patient had a medical problem that require drug therapy (an indication for drug use), but he/she did not receive a drug for that indication;

- Improper drug selection. The patient had a drug indication but he/ she used improper drug;

- Subtherapeutic dosage. The patient had a medical problem that is being treated with too little of the correct drug;

- Failure to receive drugs. The patient had a medical problem as an effect on receiving a drug (e.g., pharmaceutical, physiological, sociological, or economic effects).

- Overdosage. The patient had a medical problem that is being treated with too much of correct drug (toxicity);

- Adverse drug reactions. The patient had a medical problem as a consequence of an occurred adverse effect;

- Drug interactions. The patient had a medical problem as a consequence of drug-drug, drug-food, or drug-laboratory interactions; 
- Drug use without indication. The patient used a drug for non-valid medical valid indication.

Information on drugs, such as dosing, potential interaction, and adverse reaction, was based on the Drug Information Handbook. Stockley's Drug Interactions was used to identify drug interactions.

\section{Data collection}

Patient demographics (e.g., age, gender,), diagnosis, medical history, and medications taken before admission, clinical signs, and laboratory data were recorded. Creatinine clearance was applied to estimate renal function [5]. DRPs experienced by the patients during hospitalized were extracted from medical records, interview with the health-care team and patients.

\section{RESULTS AND DISCUSSION}

Pharmacy clinic is not a new service in hospital, but only limited hospitals have applied this service. Ideally, clinical pharmacists do routine visit to patients to improve their quality life. Therefore, they have to be able to identify, prevent, and resolve DRPs in hospitalized patient.

In this study, 62 patients were included, consisting of 55 male patients $(89 \%)$ and 7 female patients $(11 \%)$, as shown in Table 1 . A previous

Table 1: Demographic patients

\begin{tabular}{ll}
\hline Demographic & n (\%) \\
\hline Gender & \\
Female & $7(11)$ \\
Male & $55(89)$ \\
Age & \\
$0-17$ & $3(5)$ \\
$18-64$ & $50(81)$ \\
$>65$ & 9 \\
\hline
\end{tabular}

Table 2: Classification of DRPs

\begin{tabular}{ll}
\hline Classification & n (\%) \\
\hline Untreated indications & $7(9)$ \\
Improper drug selection & $9(11)$ \\
Subtherapeutic dosage & $1(1)$ \\
Failure to receive drugs & $3(4)$ \\
Overdosages & $31(38)$ \\
Adverse drug reactions & $6(7)$ \\
Drug interactions & $8(10)$ \\
Drug use without indication & $16(20)$ \\
\hline
\end{tabular}

Table 3: Most drugs in DRPs

Ranitidine
Tranexamic acid
Ceftriaxone
Cefixime
Levofloxacin

Table 4: Clinical pharmacist's recommendation

\begin{tabular}{ll}
\hline Recommendation & n (\%) \\
\hline Dose decreased & $30(37)$ \\
Drug discontinuation & $16(20)$ \\
Monitoring & $16(20)$ \\
Drug change & $8(10)$ \\
Add therapy & $7(9)$ \\
Counseling to patient & $2(2)$ \\
Dose increased & $1(1)$ \\
Refer to prescriber & $1(1)$ \\
\hline
\end{tabular}

study also confirmed that male patients dominate females [6]. The majority patients in this study were distributed in the age group of $18-64$ years $(81 \%)$ followed by 65 years $(14 \%)$ and $0-17$ years $(5 \%)$.

A total of 37 patients $(60 \%)$ in the study were considered to have DRPs and 20 patients (54\%) have more than one DRPs. We identified 81 DRPs in this study. The most common type of DRPs was overdosage (38\%), as shown in Table 2. This finding was linier with the finding from a study conducted by Prasanna et al. [7] and Castelino et al. [8], which confirmed the high dose of prescribing as the major DRPs. Yet, this finding was different with the finding from a study conducted by Samoy et al. [9] which confirmed that adverse drug reactions was the most frequent of DRPs.

Medication dosages were not adjusted for 13 patients with either renal impairment. Our judgment was based on clearance creatinin's patients using Cockcroft and Gault method. The half-life eliminations of drug were long in renal impairment patients because the drugs were excreted by renal [10]. The common drugs that account for overdosages in this study involve ranitidine, tranexamic acid, metoclopreamid, tramadol, ciprofloxacin, captopril, cotrimoxazole, and ceftazidime as shown in Table 3. This high incidence of overdosages may be related with lack of physician's knowledge about drug pharmacodynamics and pharmacokinetics. We recommended adjustment dosage depend on clearance creatinine's patients.

Drug use without indication was the second most cause of DRPs (20\%). These findings coincides with the study conducted by Alagiriswami et al. [11] and Ganachari et al. [12] where drug use without indications and improper drug selection were the most common identified DRPs. A total of 12 antibiotics were used without SIRS, clinical signs of infection, and blood or other specimen culture. For this case, we recommend to stop the antibiotics because there were no signs of infection. The other drugs, such as folic acid, allopurinol, $\mathrm{CaCO}_{3}$, and potassium supplement, were prescribed with no laboratory evidence. This was found possibly due to delaying of laboratory reports and lack of standards of treatment.

The third most common DRPs were improper drug selection (11\%). The common drugs that account for improper drug selection in this study involves antibiotics and acid secretory inhibitors. A total of 3 patients changed parenteral antibiotic to oral antibiotic but not in a same class antibiotics. A patient got antibiotic but he had Candida sp. infection based on laboratory investigation. An infective patients show no better clinical signs with meropenem. This was found may be due to the presence of resistant infection. A patient got combination PPI-ranitidine and a nausea post-operative patient got combination antacid-ranitidineondansetron. This combination should discontinue because patients did not have a gastritis history. We recommend ranitidine and ondansetron.

Drug interactions was the next type of drug-related problems identified (10\%). It differs with study conducted by Al-Hajje et al. [13], Ayalew et al. [14], and Huri and Wee [15] where drug interactions was the most frequent of DRPs. Drug interactions in the study involve drug interaction between amlodipine and simvastatin (2); captopril and allopurinol (2); levofloxacin and ondansetron (1); aspirin and clopidogrel (1); ketorolac and amlodipine (1); potassium supplement and blood infusion (1). These drug interactions had to be monitored clinical signs and laboratory reports. For example, combination of angiotensin-converting enzyme inhibitors and allopurinol may increase the risk of leucopenia and serious infection, especially in renal impairment. Patient had to be monitored for any signs of hypersensitivity (e.g., skin reactions), a low white cell count (e.g. sore throat, fever), or check differential white blood cell before adding allopurinol, then every 2 weeks during the first 3 months of treatment, and periodically [16]. AUC of 3-hydroxy-3-methylglutaryl-CoA reductase inhibitors was significantly increased by amlodipine [17].

Untreated indications (9\%) followed by adverse drug reactions (7\%), failure to receive drugs (4\%) and subtherapeutic dosages (1\%) were 
the drug-related problems identified. In this study, there were 4 actual adverse drug reactions identified. The actual adverse drug reactions were nausea (prescribed drugs were methotrexate, vincristine, cisplatin, and doxorubicin) and constipation (from patient who got ranitidine and codeine).

Drug that accounts for failure to receive drug therapy involves minocyclin, terasozin, and simvastatin. This occurrence of failure to receive drug therapy because pharmacy did not stock drug and patient's non-adherence. The last drug-related problem in this study was subtherapeutic dosages. Adult patients got salbutamol in pediatric dose.

Most of recommendations made were decreased dose (37\%) followed by drug discontinuation (20\%) and monitoring (20\%) as shown in Table 4. Other recommendation from clinical pharmacist includes drug change, add therapy, counseling to patients, dose increased, and refer to prescriber. Drug discontinuation and drug change in this study were due to no valid medical indication for the drug therapy at this time and in improper drug selection. The addition of drug was suggested in cases such as a medical condition requires the initiation of drug therapy. Monitoring suggestion was provided for drug interactions and drug were made in patients with renal impairment.

Pharmacist need to do routine visit and review patients' therapy to identify, prevent, and solve DRPs. The contribution of pharmacist in health-care team can improve adherence patients and improve patient's quality of life.

Our study has limitations. The first was a lack of standard tools to recognize drug-related problems, so we relied on clinical experience and judgment of the clinical pharmacist for identify and classify drugrelated problems. The second, our study involved patients hospitalized in urology surgery ward and had small sample size. Because of limited generalization, further research determines drug-related problems and risk factors in other populations.

\section{CONCLUSION}

The three most common identified drug-related problems are overdosage, drug use without indications, and improper drugs. The drug-related problem was a way to ensure appropriate medication use in this patient population. Routine participation of clinical pharmacists in clinical medical rounds facilitates identifying, preventing, resolving drug-related problems, and help patients achieve their goals of therapy and improve their quality of life.

\section{REFERENCES}

1. Hepler CD, Strand LM. Opportunities and responsibilities in pharmaceutical care. Am J Hosp Pharm 1990;47(3):533-43.

2. Cipolle R, Strand L, Peter M. Pharmaceutical Care. $2^{\text {nd }}$ ed. New York, NY: Mc Graw-Hill; 1998.

3. Ernst F, Grizzile A. Drug Related morbidity and mortality: Updating the cost-of-illness model. J Am Pharm Assoc 2001;41:192-9.

4. Adusumilli P, Adepu R. Drug related problems: An over view of various classification systems. Asian J Pharm Clin Res 2014;7 Suppl 4:7-10.

5. Cockcroft D, Gault M. Prediction of creatinine clearance from serum creatinine. Nephron 1976;16 Suppl 1:31-41.

6. Vijayalakshmi S, George R, James E. Clinical pharmacist's interventions on drug related problems in a tertiary care hospital. Int J Pharm Pharm Sci 2015;7 Suppl 6:401-4.

7. Prasanna D, Kumar BS, Venkataraman R, Fuloria PC. Assessment of clinical pharmacist intervention in tertiary care teaching hospital of Southern India. Asian J Pharm Clin Res 2013;6 Suppl 2:258-61.

8. Castelino R, Sathvik B, Parthasarathi G, Gurudev K, Shetty M, Narahari M. Prevalence of medication-related problems among patients with renal compromise in an Indian hospital. J Clin Pharm Ther 2011;36 Suppl 4:481-7.

9. Samoy L, Zed P, Wilbur K, Balen R, Abu-Laban R, Roberts M. Drug-related hospitalizations in a tertiary care internal medicine service of a Canadian hospital: A prospective study. Pharmacotherapy 2006;26 Suppl 11:1578-86.

10. Munar M. Drug dosing adjustments in patients with chronic. Am Fam Physician 2007;75 Suppl 10:1487-96.

11. Alagiriswami B, Ramesh M, Parthasarathi G, Basavanagowdappa H. A study of clinical pharmacist initiated changes in drug therapy in a teaching hospital. Indian J Pharm Pract 2009;1 Suppl 2:36-45.

12. Ganachari M, Kumar BM, Shashikala C, Fibrin M. Assessment of drug therapy intervention by clinical pharmacist in tertiary care hospital. Indian J Pharm Pract 2009;38 Suppl 5:361-2.

13. Al-Hajje AH, Atoui F, Awada S, Rachidi S, Zein S, Salameh P. Drugrelated problems identified by clinical pharmacist's students and pharmacist's interventions. Ann Pharm Fr 2012;70(3):169-76.

14. Ayalew MB, Megersa TN, Mengistu YT. Drug-related problems in medical wards of Tikur Anbessa specialized hospital, Ethiopia. J Res Pharm Pract 2015;4 Suppl 4:216-21.

15. Huri HZ, Wee HF. Drug related problems in Type 2 diabetes patients with hypertension: A cross-sectional retrospective study. BMC Endocr Disord 2013;13 Suppl 2:1-25.

16. Baxter IA, Davis M, Driver S, Garner R. In: Baxter K, editor. Stockley’s Drug Interactions. $9^{\text {th }}$ ed. London: Pharmaceutical Press; 2010.

17. Nishio S, Watanabe H, Kosuge K, Uchida S, Hayashi H, Ohashi K. Interaction between amlodipine and simvastatin in patients with hypercholesterolemia and hypertension. Hypertens Res 2005;28 Suppl 3:223-7. 